\title{
On the Characteristics of Ground Motion and the Improvement of the Input Mode of Complex Layered Sites
}

\author{
Hongke Pan ${ }^{a}$, Xinxin Jiang a, ${ }^{\text {** }}$ \\ ${ }^{a}$ School of Building Engineering, Хinyu University, Хinyu City 338004, China. \\ ${ }^{b}$ Earthquake Engineering Research Center, China Institute of Water Resources and Hydropower Research, Beijing 100038, China.
}

Received 28 January 2020; Accepted 05 April 2020

\begin{abstract}
It is a hot research topic to perform the dynamic interaction analysis between the engineering structure and the soil by using the time-domain method. This paper studies the seismic behaviour of the layered sites and the seismic response of the structures using the viscous-spring artificial boundary theory. The artificial boundary model of viscous-spring is initially based on homogeneous foundation. For the layered site (Foundation), the traditional homogeneous model or equivalent load input mode is not suitable, which may bring great error. By introducing the changes of coefficients and phases of reflection and transmission of seismic waves at the interface between layers, an improved method of equivalent load input mode of traditional viscous-spring artificial boundary model is proposed. This new wave model can simulate the propagation law of seismic wave in layered site more accurately, which is available for the seismic performance of engineering structure under the condition of large and complex layered site. At last, the simplified homogeneous model, the equivalent load input method and the improved layered model input method are used to study the seismic response of the engineering example. It is shown that the results calculated by the three methods are different, which shows that the homogeneous foundation model and the conventional equivalent load input method of seismic wave cannot simulate the seismic force accurately, whereas the improved wave input model can better reflect the characteristic of traveling wave in layered sites.
\end{abstract}

Keywords: Earthquake Resistance of Engineering Structures; Layered Foundation; Time Domain Analysis Method; Seismic Wave Propagation; Improvement of Input Mode.

\section{Introduction}

Complex layered sites are often encountered in the construction process of various large-scale civil, water conservancy and transportation projects. Although the effects of layered sites on the structural dynamic response have been acknowledged, there is no comprehensive understanding and design experience of it due to the complexities. Consequently, how to evaluate the dynamic response characteristics, seismic stability and seismic measures of the interaction between superstructure and soil has become a difficult problem for the builders, and sometimes even directly affects the construction of the project. This paper is devoted to the analysis of structural dynamic response in layered sites.

It has been acknowledged that the dynamic soil-structure interaction in the analysis of seismic response should be considered. Various numerical methods are developed to simulate the seismic response, and they can be inducted as global artificial boundary and local artificial boundary theory [1]. The global artificial boundary theory, typically

*Corresponding author: jiangxx_dlut@163.com

http://dx.doi.org/10.28991/cej-2020-03091512

(C) 2020 by the authors. Licensee C.E.J, Tehran, Iran. This article is an open access article distributed under the terms and conditions of the Creative Commons Attribution (CC-BY) license (http://creativecommons.org/licenses/by/4.0/). 
represented by boundary element method [2] and scaled boundary finite element method [3], can satisfy all field formulas and boundary conditions in infinite domain. The local artificial boundary theory is developed on the concept of unilateral wave, which is typical represented by viscous boundary method [4], viscous-spring artificial boundary method [5] and transmission boundary method [6]. These initial patterns of the dynamic soil-structure interaction are established with homogenous soil. Afterwards, in order to deal with more complex engineering conditions, the researches carry out experiments on complex sites and propose various methods to broaden its application. For example, Moghadam and Baziar investigated the effect of a circular subway tunnel on the ground motion amplification pattern by Shaking table testing and numerical simulation [7]. Sun et al. used the analytical study and numerical analysis to characterize the underlying soft soil layer-tunnel interaction problem [8]. Karabalis and Mohammadi used a 3-D frequency domain BEM equations in conjunction with infinite space fundamental solutions to simulate the layered soil medium [9]. Birk and Behnke derived a modified SBFEM for the analysis of 3D-layered continua based on the use of a scaling line instead of a scaling centre, and the dynamic stiffness coefficients were calculated to demonstrate the accuracy of the method [10]. Li et al. developed a time-domain method to calculate the free field motion of a layered half-space subjected to oblique incident body waves [11]. Liu and Wang developed a 1D finite element method in time domain to calculate the in-plane wave motion of free field in elastic layered space by oblique seismic incidence [12].

At present, the analysis of structure-foundation interaction using time-domain method is a hot topic, in which the viscous-spring artificial boundary performs well in the application of computational precision, stability and large-scale finite element software, and its ground motion input mode is easy to simulate the propagation process of seismic wave and the non-uniform change of field motion caused by the oblique incidence of seismic wave, so it has been widely used [12]. The viscous-spring artificial boundary theory is originally based on the homogenous soil. When it comes to complex layered sites, the major challenge is to simulate the seismic wave in the truncation boundary. It is well known that the seismic wave will be reflected and transmitted at the soil interface due to the different mechanical parameters of the soil. However, most of the existing research and engineering applications do not examine that characteristic of layered sites. One method is to take the soil parameters adjacent to the foundation as homogeneous soil model instead of the layered sites. Another method is based on layered foundation model but adopt conventional equivalent load mode of ground motion input mode, which only converts incident wave and reflected wave at the free top surface into equivalent load form and then inputs them into the system to solve the dynamic response of the whole system. For homogeneous sites, seismic waves are reflected at the free surface, and the equivalent load reflects the superposition effect of incident waves and reflected waves. For layered foundations, seismic waves will reflect and transmit at the interface of the interlayer materials, and the amplitude and phase of the reflected and transmitted waves will change [13-15]. If the conventional equivalent load input mode is still used in layered sites, it is difficult to consider the variation of the amplitude and phase of the fluctuation, which makes the fluctuation input of the artificial boundary node different from the actual fluctuation amplitude and phase of the internal node of the layered foundation model, resulting in the inconsistency of the vibration of the boundary node and the internal node of the model, thus causing relatively large calculation errors [16-18].

In order to simulate the propagation of seismic waves in a layered foundation, the input model of seismic wave under equivalent load is improved based on the viscous-spring artificial boundary model, and the reflection and transmission coefficient and phase change values of seismic waves at the material interface are introduced, so as to improve the equivalent load input mode for wave input method under layered foundation. The method inherits the advantages of viscous-spring artificial boundary.

This paper commences with the wave propagation process in a two layered site. The phenomenon of seismic reflection and transmission at the interface of the soil is illustrated, followed by derivation of formulas for reflection and transmission of seismic waves at the interface between layers. And an improved input mode of seismic waves in layered sites is proposed based on these formulas. Engineering example demonstrates the accuracy of the new seismic input mode and its implementation.

\section{Wave Characteristics of Seismic Waves in Layered Sites}

\subsection{Multiple Reflection and Transmission of Seismic Waves at Horizontal Interfaces}

The change of amplitude and phase of seismic wave propagating in layered site (foundation), showing different propagating characteristics compared with that in homogeneous foundation, is mainly caused by the different material parameters of each layer. The change of mechanical parameters at the interlayer interface makes part of the wave propagation energy reflect back to the lower soil layer, while the other part of the wave energy continues to propagate upward. Figure 1 shows the propagation process of the seismic wave vertically incident to the two-layer half-space free field. The interfaces are numbered 0,1 , and 2 from bottom to top, the interface 1 is interlayer material interface, and the interface 2 is free surface, the shear wave velocities of the first and second layers of soil are $\mathrm{C}_{\mathrm{s} 1}$ and $\mathrm{C}_{\mathrm{s} 2}$ respectively, the initial input time of seismic wave is 0 . The wave pattern of typical time is selected, and the arrow is the wave front of seismic wave. 
Interface 2

The second layer:

shear wave velocity $\mathrm{C}$ s2

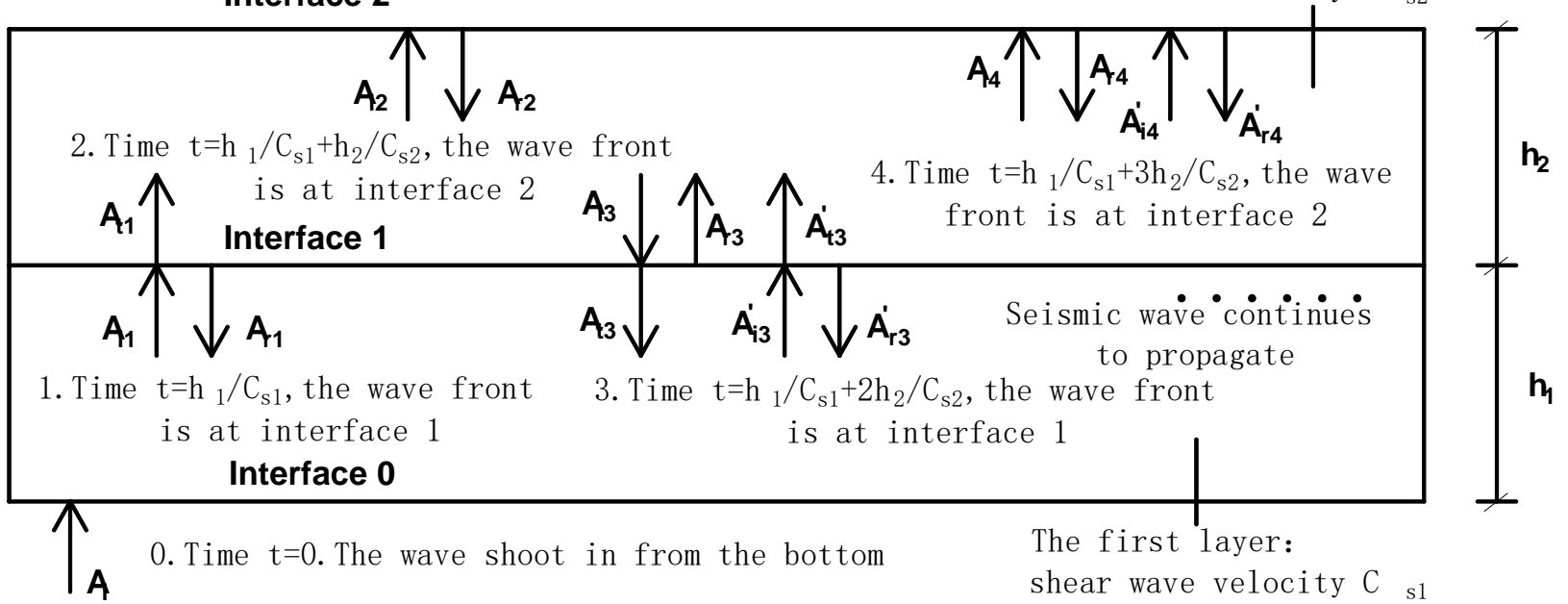

Figure 1. The sketch of seismic wave reflection and transmission on the interface

1) When $\Delta t=\frac{h_{1}}{C_{s 1}}$ : the seismic wave is reflected and transmitted at interface 1 , and the amplitudes of incident wave, reflected wave and transmitted wave are recorded as $A_{i 1}, A_{r 1}$ and $A_{t 1}$ respectively;

2) When $\Delta t=\frac{h_{1}}{C_{s 1}}+\frac{h_{2}}{C_{s 2}}$ : the seismic wave is reflected at interface 2 , and $A_{i 1}, A_{r 2}$ represent the amplitude of the incident wave and the reflected wave;

3) When $\Delta t=\frac{h_{1}}{C_{s 1}}+\frac{2 h_{2}}{C_{s 2}}$, two seismic waves are reflected and transmitted simultaneously at the interface 1. Firstly, the downward seismic waves are reflected by the top surface, $A_{i 3}, A_{r 3}$ and $A_{t 3}$ are the amplitudes of incident wave, reflected wave and transmitted wave respectively, and $A_{i 3}=A_{r 2}$. Secondly, the upward seismic waves are reflected by the ground, similarly, $A_{i 3}^{\prime}, A_{r 3}^{\prime}$ and $A_{t 3}^{\prime}$ are the amplitudes of incident wave, reflected wave and transmitted wave respectively;

4) $\Delta t=\frac{h_{1}}{C_{s 1}}+\frac{3 h_{2}}{C_{s 2}}$ : two waves propagating upward on the top surface with the amplitude $A_{r 3}$ and $A_{t 3}^{\prime}$ are reflected simultaneously. The phases change after reflection;

5) As shown in the previous four steps, the seismic waves will be reflected and transmitted continuously at interface 1 and 2 during the propagation process. The seismic waves in the foundation are superimposed on each other, and some wave forms will persist in the soil layer.

\subsection{Equations for Reflection and Transmission of Seismic Waves at the Interface between Layers}

As shown in Figure 2, the seismic wave is obliquely incident at the horizontal interface, and reflection and transmission occur. Snell's theorem states that the various waves in the wave system on the interface have the same apparent propagation velocity along the interface, and the corresponding mathematical expression is expressed as:

$\frac{\sin \theta_{i s h}}{C_{s 1}}=\frac{\sin \theta_{r s h}}{C_{s 1}}=\frac{\sin \theta_{t s h}}{C_{s 2}}$

With

$\theta_{i s h}=\theta_{r s h}$

The incident wave, reflected wave and transmitted wave in Figure 2 are respectively represented by subscripts $i$, $r$ and $\mathrm{t}$; A represents the amplitude of the wave; $\theta$ is the angle between the direction of wave propagation and the interface normal. 


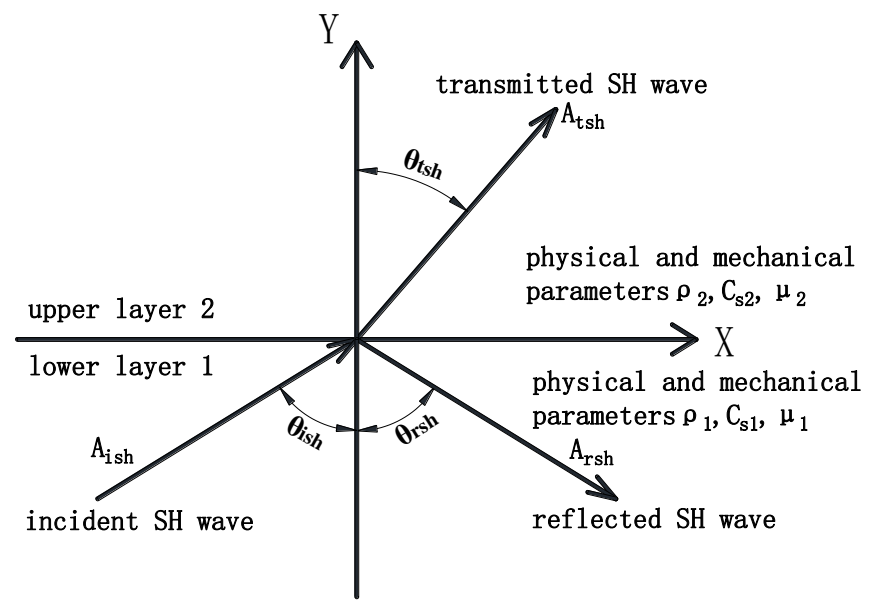

Figure 2. The sketch map of reflection and transmission of the SH wave on the interface

The relationship between the amplitude of incident $\mathrm{SH}$ wave, reflected $\mathrm{SH}$ wave and transmitted SH wave is derived below. Assuming that the material is in close contact at the interface, the equations for the boundary conditions at the interface 1 are obtained by the compatibility and equilibrium at the interface.

$U^{(1)}=U^{(2)}$

$\tau_{x y}^{(1)}=-\tau_{x y}^{(2)}$

Where $\mathrm{U}$ is the horizontal displacement; $\tau$ is the shear stress; the superscript 1 and 2 denote the lower soil layer 1 and the upper soil layer 2 , respectively.

$U_{i s h}, U_{r s h}$ and $U_{t s h}$ are assumed respectively (only Y-direction deformation occurs).

$U_{i s h}=A_{i s h} \exp \left[i \omega\left(t-\frac{\cos \theta_{i s h}}{C_{s 1}} y\right)\right]$

$U_{r s h}=A_{r s h} \exp \left[i \omega\left(t-\frac{\cos \theta_{r s h}}{C_{s 1}} y\right)\right]$

$U_{t s h}=A_{t s h} \exp \left[i \omega\left(t-\frac{\cos \theta_{t s h}}{C_{s 2}} y\right)\right]$

The displacements of soil layer 1 and 2 in Figure 2 are respectively:

$U^{(1)}=U_{i s h}^{(1)}+U_{r s h}^{(1)}$

$U^{(2)}=U_{t s h}$

In the above formulas, $\tau_{y x}^{(1)}, \tau_{y x}^{(2)}$ can be derived from the linear elastic stress-strain relation $\tau=G \frac{\partial u}{\partial y}$. Substituting Equations 8 and 9 and the derived $\tau_{y x}^{(1)}, \tau_{y x}^{(2)}$ into the boundary conditions of Equations 3 and 4 , yields:

$1+\frac{A_{r s h}}{A_{i s h}}=\frac{A_{t s h}}{A_{i s h}}$

$-\rho_{1} C_{s 1} \cos \theta_{i s H}+\rho_{1} C_{s 1} \cos \theta_{r s h} \frac{A_{r s h}}{A_{i s h}}=-\rho_{2} C_{s 2} \cos \theta_{t s h} \cos \theta_{t s H} \frac{A_{t s h}}{A_{i s h}}$

Substituting Equation 10 into 11, and noting that $\theta_{i s h}=\theta_{r s h}$, the seismic reflection coefficient and transmission coefficient formulas are expressed as:

$\frac{A_{r s h}}{A_{i s h}}=\frac{\rho_{1} C_{s 1} \cos \theta_{i s h}-\rho_{2} C_{s 2} \cos \theta_{t s h}}{\rho_{1} C_{s 1} \cos \theta_{i s h}+\rho_{2} C_{s 2} \cos \theta_{t s h}}$

$\frac{A_{t s h}}{A_{i s h}}=\frac{2 \rho_{1} C_{s 1} \cos \theta_{i s h}}{\rho_{1} C_{s 1} \cos \theta_{i s h}+\rho_{2} C_{s 2} \cos \theta_{t s h}}$ 
Typically, when the incident wave is perpendicular to the interface, $\theta_{i s h}=90^{\circ}$. Substituting $\alpha=\frac{\rho_{2} C_{s 2}}{\rho_{1} C_{s 1}}$ into Equations 12 and 13, they are simplified as:

$\frac{A_{\text {ish }}}{A_{i s h}}=\frac{1-\alpha}{1+\alpha}$

$\frac{A_{i s h}}{A_{i s h}}=\frac{2}{1+\alpha}$

If the interface is a free surface, then: $\alpha=0, \frac{A_{r s h}}{A_{i s h}}=1$.

$\mathrm{P}$ wave and SV wave can be derived similarly when they are incident, but it should be noted that there will be waveform conversion at the interface of $\mathrm{P}$ wave and $\mathrm{SV}$ wave oblique incidence, that is, $\mathrm{SV}$ wave and $\mathrm{P}$ wave exist in both reflected wave and transmitted wave.

\section{Input Mode of Seismic Waves in Layered Sites}

\subsection{Improved Wave Input Mode of Equivalent Load Form}

The conventional expression of the equivalent load of the traditional viscous-spring artificial boundary is express as [18]:

$F_{B}(t)=\tau_{0}\left(x_{B}, y_{B}, t\right)+C_{B} \omega_{0}\left(x_{B}, y_{B}, t\right)+K_{B} \omega_{0}\left(x_{B}, y_{B}, t\right)$

Where, $x_{B}, y_{B}$ and $t$ are the coordinates and time of point $\mathrm{B}$ of the artificial boundary successively; $\omega_{0}\left(x_{B}, y_{B}, t\right)$ stands for the displacement and velocity of the incident wave field at the node; $C_{B}$ and $K_{B}$ are the damper coefficient and the spring elasticity coefficient, respectively.

The seismic wave of layered foundation is reflected and transmitted at the interface between layers, and the displacement amplitude and phase change. Displacement $\omega_{r}\left(x_{B}, y_{B}, t\right)$ of the reflected wave and displacement $\omega_{t}\left(x_{B}, y_{B}, t\right)$ of the transmitted wave are expressed as:

$$
\begin{aligned}
& \omega_{r}\left(x_{B}, y_{B}, t\right)=\frac{A_{r}}{A_{i}} \omega_{0}\left(x_{B}, y_{B}, t\right) \\
& \omega_{t}\left(x_{B}, y_{B}, t\right)=\frac{A_{t}}{A_{i}} \omega_{0}\left(x_{B}, y_{B}, t\right)
\end{aligned}
$$

After derivation of Equations 17 and 18, the corresponding expression of node velocity can be obtained, and then substituting it into Equation 16, the expression $F^{\prime}{ }_{B}(t)$ of the improved equivalent load of reflection and transmission of seismic wave at the interface is obtained:

$$
F_{B}^{\prime}(t)=\tau_{0}\left(x_{B}, y_{B}, t\right)+C_{B}\left[\frac{A_{t}}{A_{i}} \omega_{0}\left(x_{B}, y_{B}, t\right)-\frac{A_{r}}{A_{i}} \omega_{0}\left(x_{B}, y_{B}, t\right)\right]+K_{B}\left[\frac{A_{t}}{A_{i}} \omega_{0}\left(x_{B}, y_{B}, t\right)+\frac{A_{r}}{A_{i}} \omega_{0}\left(x_{B}, y_{B}, t\right)\right]
$$

If the physical and mechanical parameters of the upper and lower layers of the interface are the same, the layered foundation will be degraded into a homogeneous site. $\frac{A_{t}}{A_{i}}=1, \frac{A_{r}}{A_{i}}=0$. It means that seismic waves at interlayer interface do not reflect, and the improved expression (19) of equivalent load can be changed to (16), which indirectly verifies the correctness of the derivation process.

\subsection{Realization of Wave Input of Layered Site in ANSYS}

In Software ANSYS, the wave input of viscous-spring artificial boundary of layered foundation is redeveloped, and then the macro file is made to generate ANSYS tool button, which can automatically load the input file of seismic wave and the viscous-spring artificial boundary for seismic response analysis. It includes spring damper application module, equivalent load generation and loading module, solver solution and post-processing module.

1) Automatic application of spring damper: The boundary of the foundation is a box-shaped artificial boundary, which can automatically search for the boundary nodes and store them. The spring damper of the boundary nodes can be applied automatically by "Ndnext" command.

2) Generation and application of equivalent load: In the process of simulating multiple reflection and transmission of seismic waves, two parameters of two-dimensional array can be used to track the position of seismic wave front and the number of times of transmission and reflection respectively, so as to express the delay phase of seismic 
waves and the amplitude after multiple reflection and transmission. For each solution time, the equivalent load on the node after each wave superposition is calculated and applied to the boundary node for solution, which ensures the accurate simulation of wave input at each time.

3) Solver solution and post-processing: The Newmark integral is used to solve the dynamic response in ANSYS transient analysis, and linear elastic solution is used in this paper. Post-processing mainly uses Post1 and Post26 to extract stress, displacement, acceleration and draw contours.

\section{Engineering Example}

A roller compacted concrete gravity dam located on two layers of foundation is chosen to illustrate actual engineering situation that can be modelled accurately by applying the improved seismic input mode. The analysis of the free field of layered site showed that the seismic behaviour of the layered foundation was significantly different from the homogenous foundation [12]. Next, the engineering adaptability of the gravity dam model is verified by the seismic response analysis of the model applied to the two-story site.

\subsection{Basic Information}

The dam is a roller compacted concrete gravity dam located on two layers of foundation. The height of the dam body is $73 \mathrm{~m}$, the top thickness of the dam is $6 \mathrm{~m}$, and the slope of the downstream dam is 1:0.7. There are two types of materials for dam concrete: the dynamic elastic modulus of concrete outside the dam is $36.4 \mathrm{GP}_{\mathrm{a}}$, the density is $2400 \mathrm{~kg} / \mathrm{m}^{3}$, and the Poisson ratio is 0.167 ; while the dynamic elastic modulus of concrete inside the dam is $28.6 \mathrm{GP}_{\mathrm{a}}$, the density is $2400 \mathrm{~kg} / \mathrm{m}^{3}$, and the Poisson ratio is 0.167 . There are two types of materials for foundation from bottom to top: the dynamic elastic modulus at the bottom is $52 \mathrm{GP}_{\mathrm{a}}$, density is $2700 \mathrm{~kg} / \mathrm{m}^{3}$, Poisson ratio is 0.25 ; while the dynamic elastic modulus at the top is $26 \mathrm{GP}_{\mathrm{a}}$, density is $2700 \mathrm{~kg} / \mathrm{m}^{3}$, Poisson ratio is 0.25 . The designed peak ground motion acceleration at the dam site is $0.374 \mathrm{~g}$.

\subsection{Finite Element Model of Gravity Dam-Layered Site}

In the finite element model of gravity dam-layered site shown in Figure 3, the dam body is divided into grids by Plane42 unit, which is set to the plane strain problem. A total of 3164 units and 7778 nodes are divided. The viscousspring artificial boundary is simulated by spring damper, which is applied automatically by node number. The scattering source is located at the centroid of the gravity dam, and the spring coefficient R of each boundary spring damper is taken as the average value of the distance from the scattering source to each boundary surface and to the corner of the boundary. The spring damper coefficient at the material interface is taken as the average value of the upper and lower layers.

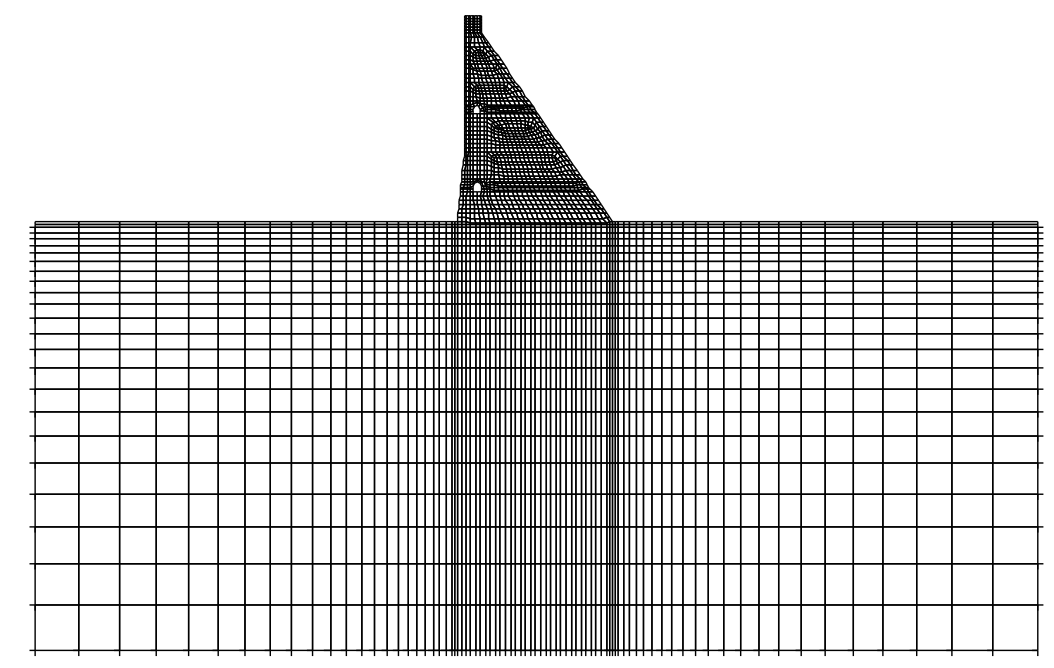

Figure 3. The finite element model of gravity dam and foundation

\subsection{Seismic Wave Processing}

The designed peak ground motion acceleration of the site where the dam is located is $0.374 \mathrm{~g}$. The target response spectrum is determined by referring to the Specifications for Seismic Design of Hydraulic Structures DL5073-2000 [19], and then the artificial seismic wave is synthesized. Wherein, the representative value $\beta_{\max }$ of the maximum design response spectrum is 2.0 for gravity dams and $20 \%$ for the lower limit of design response spectrum, i.e. 0.4 for maximum representative value $\beta_{\min }$, the site should be determined as category 1 according to the foundation 
parameters, and the characteristic period $T_{g}$ is $0.20 \mathrm{~s}$, thus the design response spectrum is determined, and the artificial seismic wave is synthesized according to the response spectrum, and the duration of the artificial seismic wave is $28 \mathrm{~s}$, as shown in Figure 4.

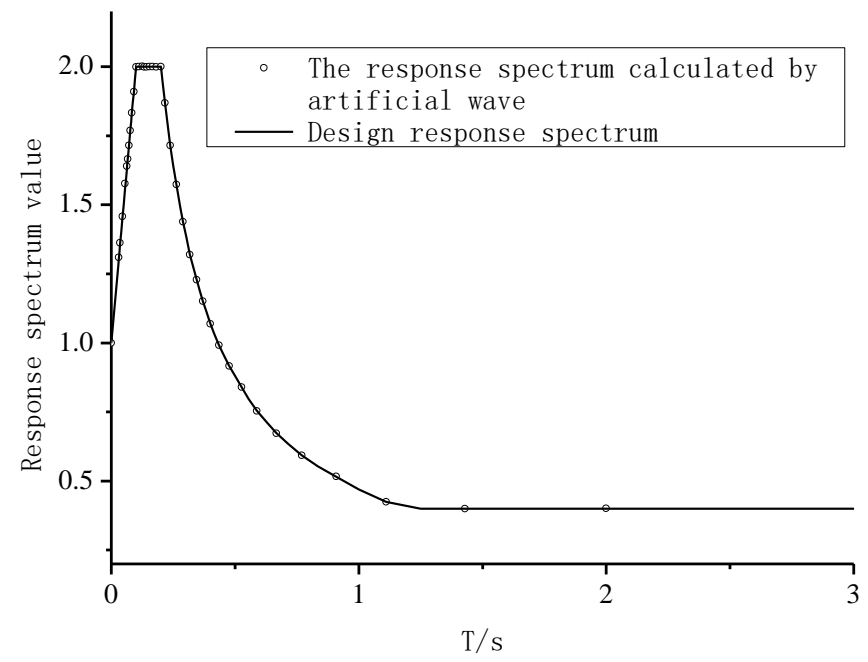

A. Comparison between calculation response spectrum and design response spectrum

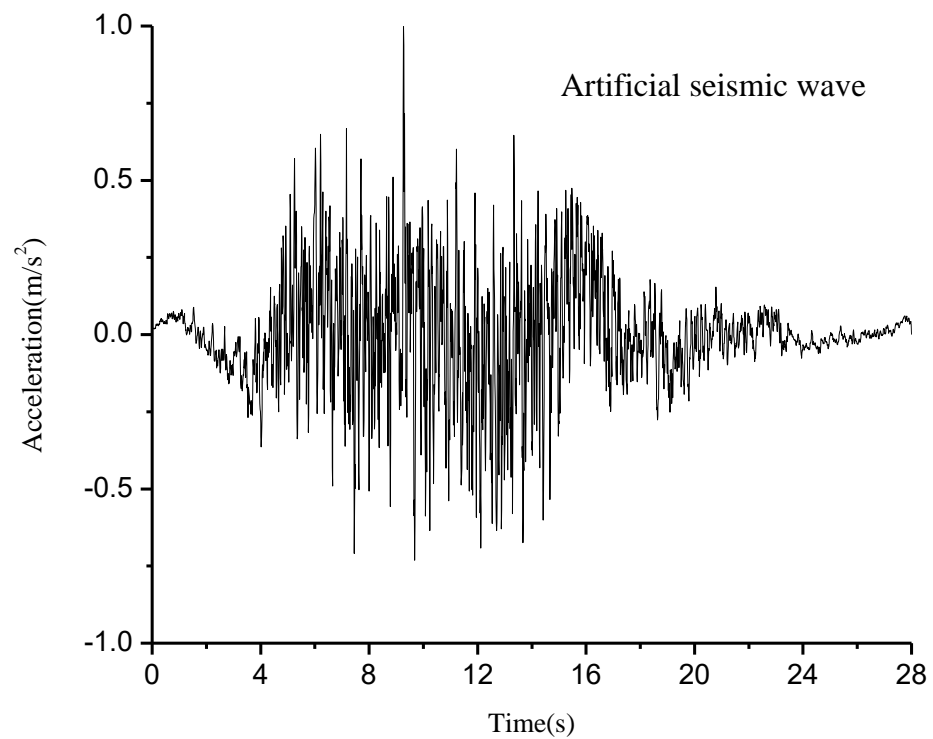

B. Acceleration time history of artificial seismic waves

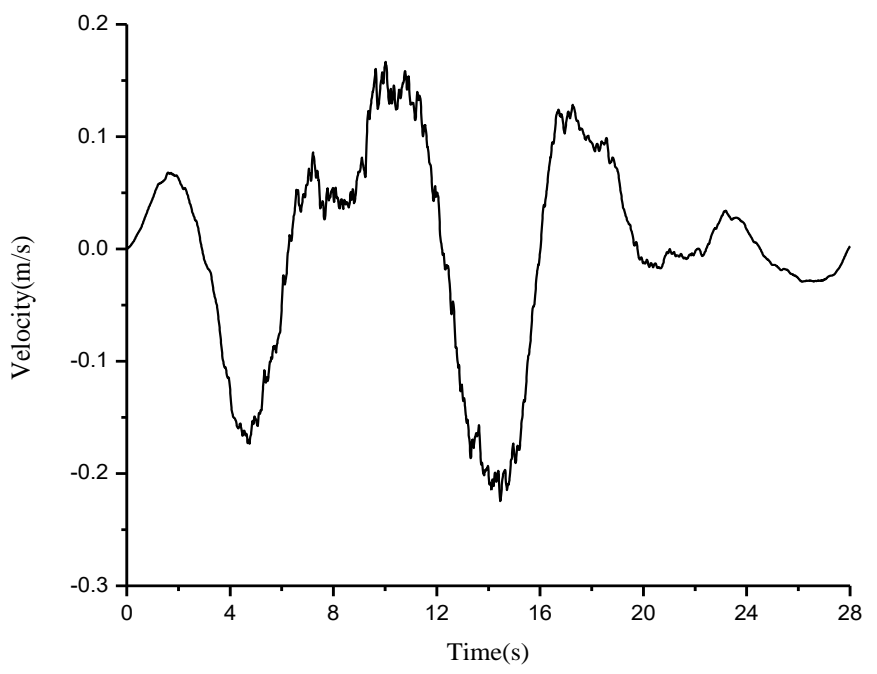

C. Velocity time history of artificial seismic waves 


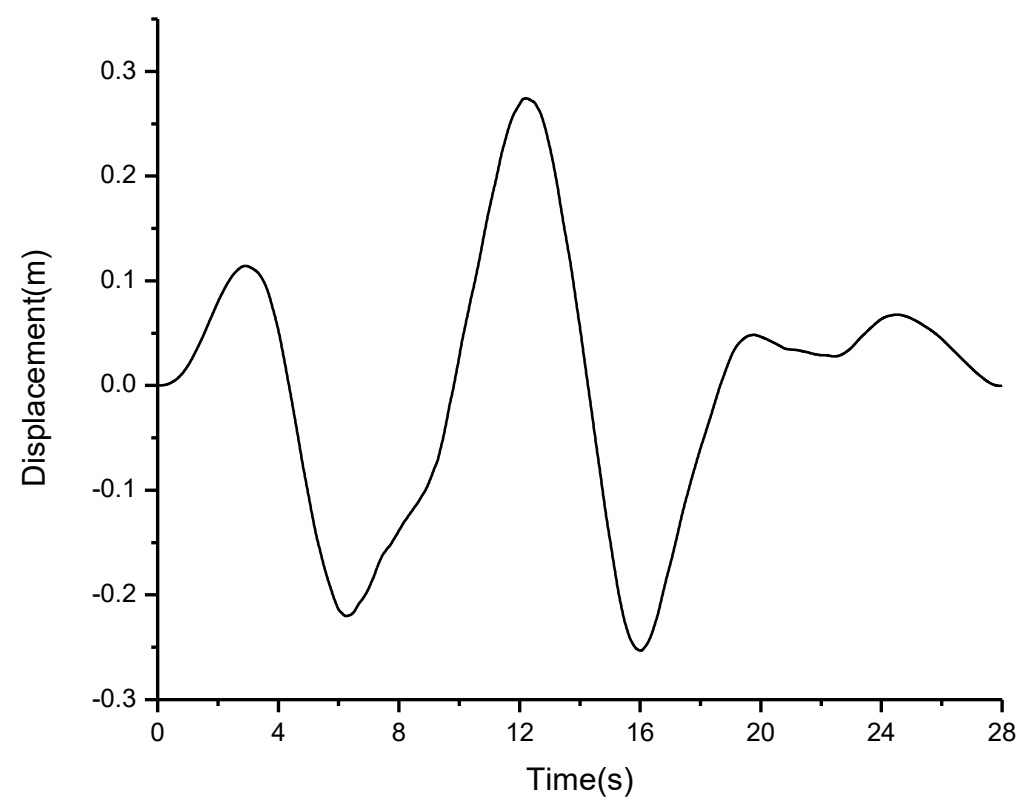

D. Displacement time history of artificial seismic waves

Figure 4. The Artificial Seismic Wave

\subsection{Calculation Conditions}

The seismic response calculation method considering layered foundation structure at traditional viscous-spring artificial boundary is compared with the viscous-spring artificial boundary model and wave input method considering seismic wave reflection and transmission at material interface in this paper, and three calculation conditions are designed respectively, as shown in Table 1. Only the horizontal shear wave of seismic wave is input to calculate the response of empty reservoir without water under the horizontal earthquake.

Table 1. The model and mechanical parameters of three methods

\begin{tabular}{|c|c|c|c|}
\hline & Scheme 1 (Case 1) & Scheme 2 & Scheme 3 \\
\hline Model description & Simplified homogeneous model & $\begin{array}{l}\text { Layered site model (Equivalent load inp } \\
\text { ut mode of seismic wave) }\end{array}$ & $\begin{array}{l}\text { Layered site model (Improved input mode } \\
\text { of seismic wave) }\end{array}$ \\
\hline Stratum parameter & $\begin{array}{l}\text { Foundation density is } 2700 \mathrm{~kg} / \mathrm{m}^{3} \text {, } \\
\text { elastic modulus is } 26 \mathrm{GP}_{\mathrm{a}} \text {, Poisson } \\
\text { ratio is } 0.167 .\end{array}$ & $\begin{array}{l}\text { The density of upper foundation is } 2700 \\
\mathrm{~kg} / \mathrm{m}^{3} \text {, the elastic modulus is } 26 \mathrm{GPa} \text {, an } \\
\mathrm{d} \text { Poisson ratio is } 0.167 \text {; the density of th } \\
\text { e lower foundation is } 2700 \mathrm{~kg} / \mathrm{m}^{3} \text {, the ela } \\
\text { stic modulus is } 52 \mathrm{GPa} \text {, and the Poisson } \\
\text { ratio is } 0.167 \text {. }\end{array}$ & $\begin{array}{l}\text { The density of upper foundation is } 2700 \mathrm{k} \\
\mathrm{g} / \mathrm{m}^{3} \text {, the elastic modulus is } 26 \mathrm{GPa} \text {, and } \mathrm{P} \\
\text { oisson ratio is } 0.167 \text {; the density of the lo } \\
\text { wer foundation is } 2700 \mathrm{~kg} / \mathrm{m}^{3} \text {, the elastic } \\
\text { modulus is } 52 \mathrm{GPa} \text {, and the Poisson ratio i } \\
\mathrm{s} 0.167 \text {. }\end{array}$ \\
\hline Model characteristics & $\begin{array}{l}\text { The homogeneous foundation has } \\
\text { no reflection and transmission of } \mathrm{s} \\
\text { eismic waves at the interface. }\end{array}$ & $\begin{array}{l}\text { The wave input does not take into accou } \\
\text { nt the reflection and transmission of seis } \\
\text { mic waves at the interface. }\end{array}$ & $\begin{array}{l}\text { The wave input takes into account the refl } \\
\text { ection and transmission of seismic waves } \\
\text { at the interface. }\end{array}$ \\
\hline
\end{tabular}

\subsection{Arrangement of Calculation Results}

\subsubsection{Calculation results of displacement and acceleration}

The results of displacement and acceleration calculation are shown in Figure 5. 


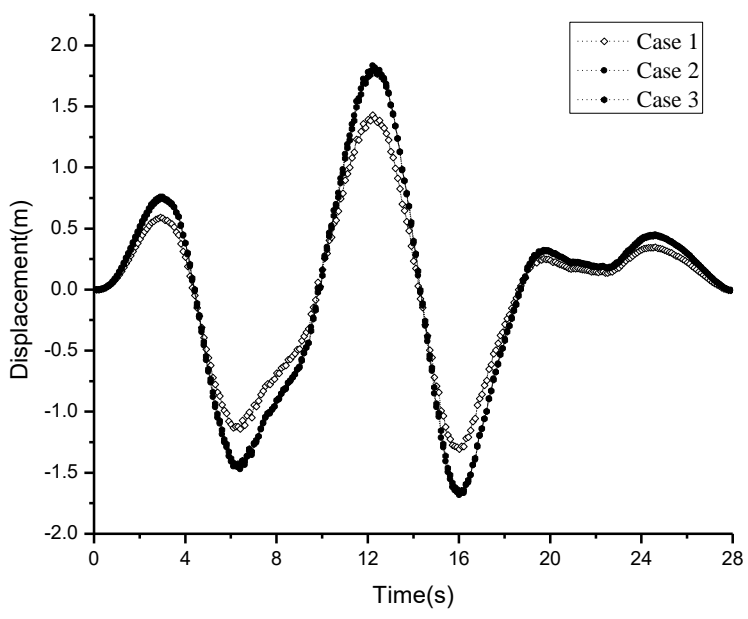

A. Contrast diagram of dam top displacement under three working conditions

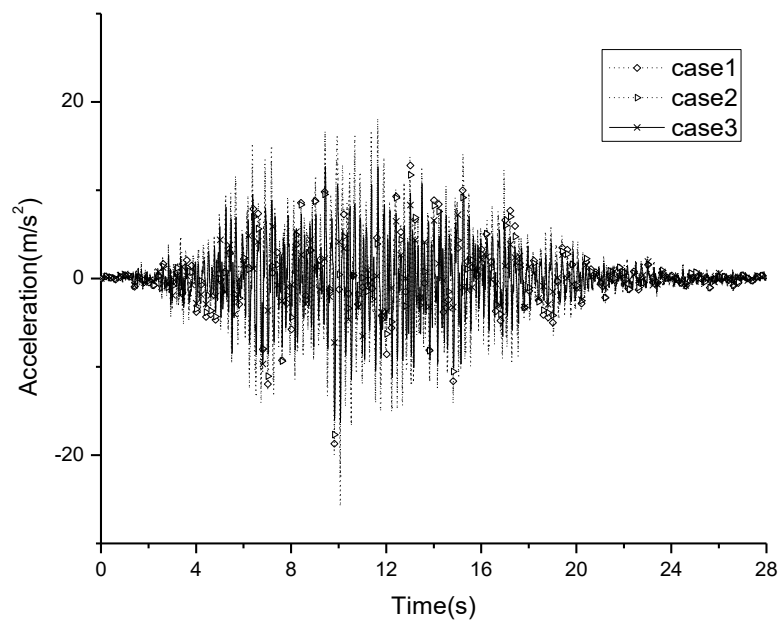

B. Contrast diagram of acceleration of dam top under three working conditions

Figure 5. The top results of displacement and acceleration

\subsubsection{Calculation Results of Stress Diagram}

The stress diagrams are shown in Figures 6 to 8 .

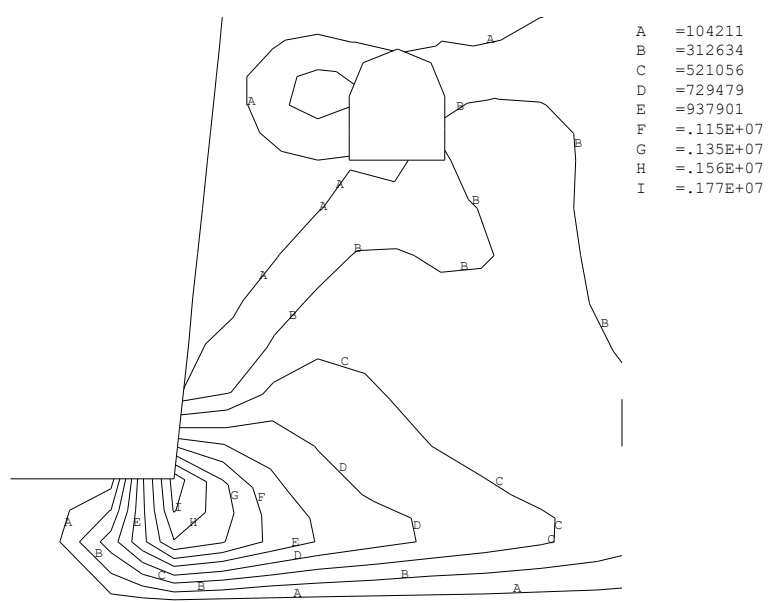

A. Isogram of local vertical stress in dam heel

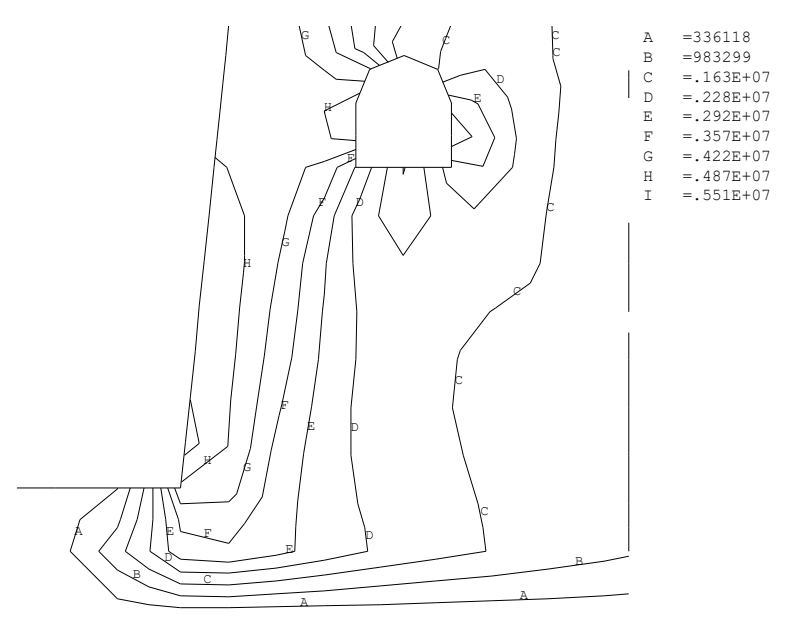

B. Isogram of local first principal stress at dam heel

Figure 6. The results of the scheme 1 


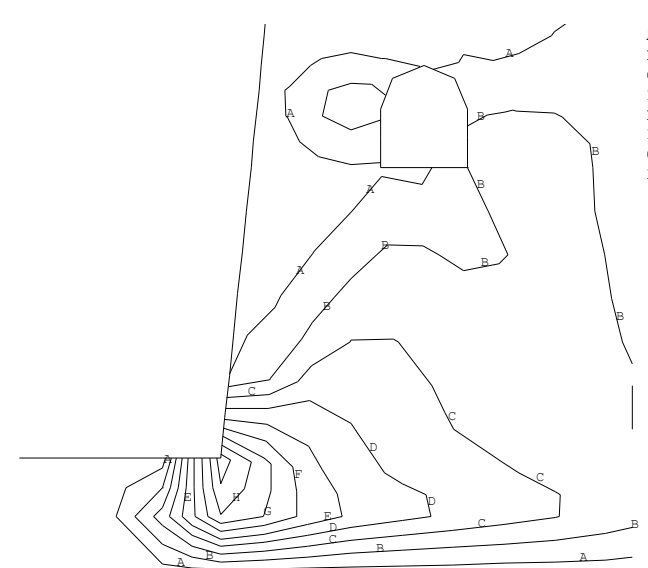

A. Isogram of local vertical stress in dam heel

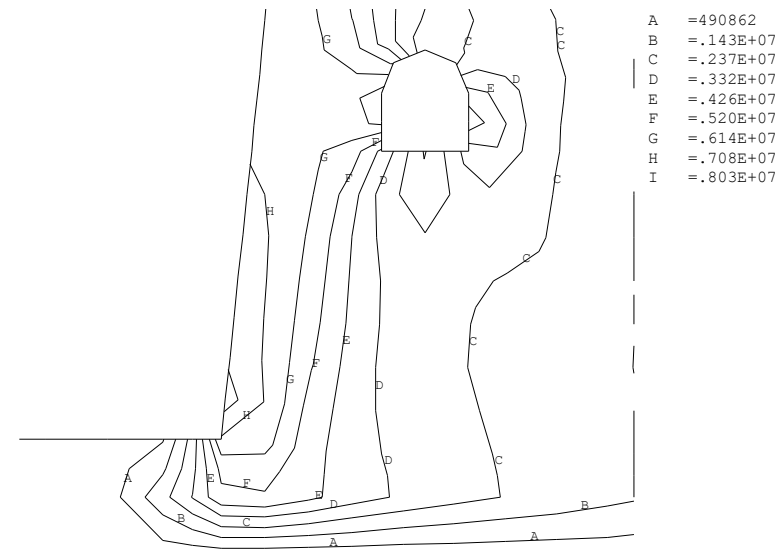

B. Isogram of local first principal stress at dam heel

Figure 7. The results of the scheme 2

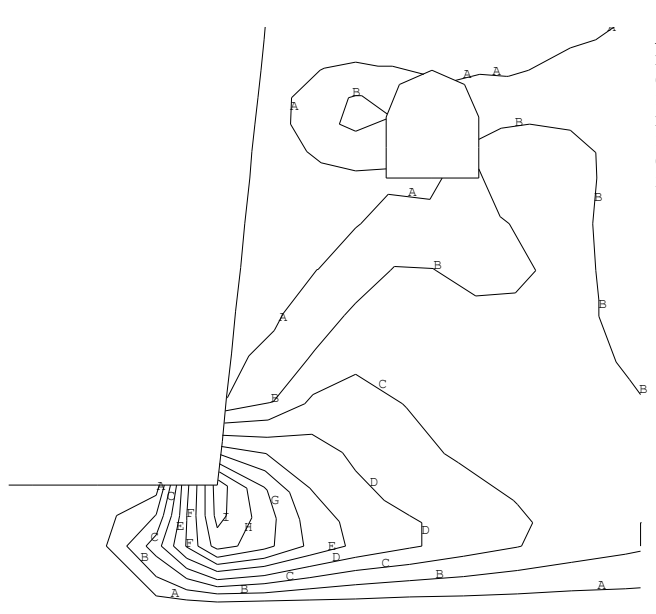

A. Isogram of local vertical stress in dam heel

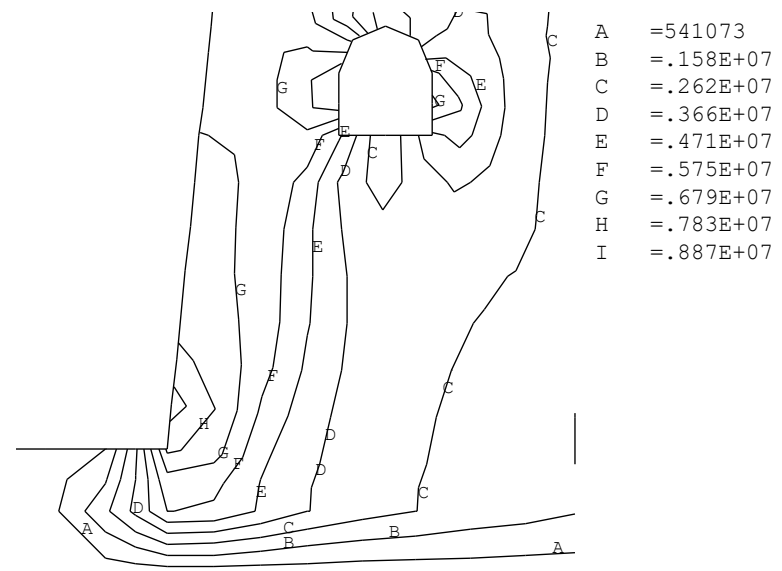

B. Isogram of local first principal stress at dam heel

Figure 8. The results of the scheme 3

\subsection{Results and Discussion}

Figure 5 plots the displacement and acceleration time history of the extracted dam crest joints. The displacement amplitude in scheme one is $1.37 \mathrm{~m}$, and that of scheme two is approximately the same as that of scheme three, which is $1.74 \mathrm{~m}$, indicating that the calculation result of layered foundation is smaller when it is simplified into homogeneous foundation. The results of acceleration calculation also illustrate this problem.

The exact stress distribution of the local stress in the heel of the dam under the three schemes is also plotted in Figures 6 to 8. The local vertical stress distribution is basically the same as the isogram of the first principal stress, but the values are obviously different, which are successively scheme 3, scheme 2 and scheme 1 from the largest to the smallest. The stresses in the heel of the dam of scheme 2 differ greatly from that of scheme 3 , in which the relative error of vertical stress is $37.2 \%$, and the relative error of principal stress is $10.5 \%$. Combined with Figures 6 to 8 , it can be seen that although the displacement and acceleration of the dam crest calculated in Scheme 2 are similar to those in Scheme 3, the significant difference in the results of heel stress calculation illustrates the necessity of considering the wave input method of reflection and transmission of seismic wave in Scheme 3 , because the wave input of boundary node is inconsistent with the vibration of internal node in Scheme 2, which restricts the propagation of real seismic wave. At the same time, the calculation results show that, for such gravity concrete dam, the stress at the heel of gravity dam is the key part of earthquake resistance, which needs to be paid attention to in the seismic design and construction of gravity dam.

In this example, the presented results show that: for the layered site, when the physical and mechanical parameters of the soil layers are quite different, the model of simplified homogeneous foundation may bring great errors; similarly, when the layered foundation model is adopted, the conventional equivalent load input mode of seismic wave also cause errors compared with the improved input mode of seismic wave. The traditional equivalent load input mode 
is mainly applicable to the case of homogeneous foundation at first, which only considers the superposition effect of incident wave and reflected wave of seismic wave at top free surface. However, in the actual layered foundation, seismic wave will be reflected and transmitted at the interface between layers (and may occur continuous reflection and transmission phenomenon). Therefore, this paper proposes to consider the reflection and transmission coefficients and phase changes of seismic wave at the interlayer interface, so as to reflect the actual propagation law and mode of seismic wave.

\section{Conclusion}

This paper study the seismic behavior of layered sites and has developed an improved seismic input mode of the viscous-spring artificial boundary theory based on the characteristic of traveling wave. For the seismic response analysis of layered site, its dynamic characteristics have an important influence on the structural dynamic response. When viscous-spring artificial boundary is used to simulate the dynamic interaction between the structure and the foundation, the homogeneous foundation model is difficult to reflect the propagation characteristics of seismic waves between the soil layers. At present, the most widely used method is the layered site model - the equivalent load input mode of seismic waves. However, engineering experience and theoretical analysis show that the traditional wave input mode used in layered site condition may lead to the inconsistency between the wave input of the boundary node and the vibration of the internal node, thus causing the calculation error. Considering the reflection and transmission of seismic wave on the layered interface, the improved method can better reflect the actual situation of the site and improve the accuracy of the calculation results.

Compared with the conventional equivalent load input mode, the improved wave input mode continuously tracks the propagation process of seismic wave, keeps the consistency between the boundary input and the internal node vibration, thus expanding the application scope of the original viscous-spring artificial boundary model. In addition, in the author's further study; it has been preliminarily found that whether it is necessary to adopt the improved seismic wave input mode method for layered foundation is also closely related to the physical quantities such as the shear modulus of the stratum and the density of soil. Only when these physical quantities between the soil layers reach a certain difference, the improved seismic wave input mode should be considered. The specific difference of these physical quantities needs further study.

\section{Funding}

The research described in this paper was financially supported by science and technology research project of Jiangxi Provincial Education Department (Grant No. GJJ171061).

\section{Conflicts of Interest}

The authors declare no conflict of interest.

\section{References}

[1] Gazetas, George. “Analysis of Machine Foundation Vibrations: State of the Art.” International Journal of Soil Dynamics and Earthquake Engineering 2, no. 1 (January 1983): 2-42. doi:10.1016/0261-7277(83)90025-6.

[2] Luamba, Endi Samba, and João Batista de Paiva. "A 3D BEM/FEM Formulation for the Static Analysis of Piled Rafts and Capped Pile Groups Subjected to Vertical and Horizontal Loads." Engineering Analysis with Boundary Elements 103 (June 2019): 66-79. doi:10.1016/j.enganabound.2019.02.009.

[3] Chen, Denghong, and Shangqiu Dai. "Dynamic Fracture Analysis of the Soil-Structure Interaction System Using the Scaled Boundary Finite Element Method." Engineering Analysis with Boundary Elements 77 (April 2017): 26-35. doi:10.1016/j.enganabound.2017.01.002.

[4] Miura, Fusanori, and Hiroshi Okinaka. "Dynamic Analysis Method for 3-D Soil-Structure Interaction Systems with the Viscous Boundary Based on the Principle of Virtual Work." Doboku Gakkai Ronbunshu no. 404 (1989): $395-404$. doi:10.2208/jscej.1989.404_395.

[5] Wang, Duguo, Peixin Shi, and Chenggang Zhao. "Two-Dimensional in-Plane Seismic Response of Long-Span Bridges Under Oblique P-Wave Incidence.” Bulletin of Earthquake Engineering 17, no. 9 (June 13, 2019): 5073-5099. doi:10.1007/s10518019-00664-7.

[6] Aydinoğlu, M.Nuray. "Consistent Formulation of Direct and Substructure Methods in Nonlinear Soil-Structure Interaction." Soil Dynamics and Earthquake Engineering 12, no. 7 (1993): 403-410. doi:10.1016/0267-7261(93)90003-a.

[7] Rabeti Moghadam, Masoud, and Mohammad Hassan Baziar. "Seismic Ground Motion Amplification Pattern Induced by a Subway Tunnel: Shaking Table Testing and Numerical Simulation.” Soil Dynamics and Earthquake Engineering 83 (April 2016): 81-97. doi:10.1016/j.soildyn.2016.01.002. 
[8] Sun, Qiangqiang, Daniel Dias, and Luis Ribeiro e Sousa. "Impact of an Underlying Soft Soil Layer on Tunnel Lining in Seismic Conditions.” Tunnelling and Underground Space Technology 90 (August 2019): 293-308. doi:10.1016/j.tust.2019.05.011.

[9] Karabalis, Dimitris L., and Mohsen Mohammadi. “3-D Dynamic Foundation-Soil-Foundation Interaction on Layered Soil.” Soil Dynamics and Earthquake Engineering 17, no. 3 (January 1998): 139-152. doi:10.1016/s0267-7261(97)00047-x.

[10] Birk, C., and R. Behnke. "A Modified Scaled Boundary Finite Element Method for Three-Dimensional Dynamic SoilStructure Interaction in Layered Soil.” International Journal for Numerical Methods in Engineering 89, no. 3 (August 4, 2011): 371-402. doi:10.1002/nme.3251.

[11] Li, Shanyou, Haiyan Zhu, and Shufang Gao. "Time Domain Method for Calculating Free Field Motion of a Layered HalfSpace Subjected to Obliquely Incident Body Waves.” Earthquake Engineering and Engineering Vibration 6, no. 2 (June 2007): 191-196. doi:10.1007/s11803-007-0735-1.

[12] Liu, Jing-bo, and Yan Wang. "A 1D time-domain method for in-plane wave motion of free field in layered media." Engineering Mechanics 24, no. 7 (2007): 16-22.

[13] Pasqua, Fernando Della, Rafael Benites, Chris Massey, and Mauri MacSaveney. "Numerical Evaluation of 2D Versus 3D Simulations for Seismic Slope Stability." Advancing Culture of Living with Landslides (2017): 557-564. doi:10.1007/978-3319-53498-5_64.

[14] Maercklin, Nils, and Aldo Zollo. "Estimation of Elastic Contrasts in a Layered Model from seismicPS-to-PPamplitude Ratios." Geophysical Journal International 179, no. 3 (December 2009): 1617-1626. doi:10.1111/j.1365-246x.2009.04350.x.

[15] Riga, Evi, Konstantia Makra, and Kyriazis Pitilakis. "Investigation of the Effects of Sediments Inhomogeneity and Nonlinearity on Aggravation Factors for Sedimentary Basins.” Soil Dynamics and Earthquake Engineering 110 (July 2018): 284-299. doi:10.1016/j.soildyn.2018.01.016.

[16] Majhi, S., P.C. Pal, and S. Kumar. "Propagation of SH Waves in a Visco-Elastic Layer Overlying an Inhomogeneous Isotropic Half-Space.” Ain Shams Engineering Journal 9, no. 4 (December 2018): 675-680. doi:10.1016/j.asej.2016.03.011.

[17] Ga, Lin, Han Zejun, and Li Weidong. "A precise integration approach for the dynamic-stiffness matrix of strip footings on a layered medium." Chinese Journal of Theoretical and Applied Mechanics 44, no. 3 (2012): 557-567. doi:10.6052/0459-18792012-3-20120312.

[18] Zhao, Mi, Houquan Yin, Xiuli Du, Jingbo Liu, and Lingyu Liang. "1D Finite Element Artificial Boundary Method for Layered Half Space Site Response from Obliquely Incident Earthquake.” Earthquakes and Structures 9, no. 1 (July 25, 2015): $173-194$. doi:10.12989/eas.2015.9.1.173.

[19] The State Economic and Trade Commission of the People's Republic of China. "The Specifications for Seismic Design of Hydraulic Structures (DL5073-2000)", 2000. 